\title{
Solitary Metastatic Tumor Within the Optic Chiasm -Case Report-
}

\author{
Atsushi ARAI, Akitsugu MORISHITA*, Yusei HANADA*, and Hideo AiHARA \\ Department of Neurosurgery, Kobe University Graduate School of Medicine, Kobe, Hyogo; \\ * Department of Neurosurgery, Toyooka Hospital, Toyooka, Hyogo
}

\begin{abstract}
A 61-year-old female, previously treated for lung cancer, presented with a rare case of metastasis directly to the optic chiasm manifesting as visual deficits. Magnetic resonance imaging revealed a suprasellar mass similar to an optic glioma. At surgery, the optic chiasm appeared swollen with hypervascularity over the surface. The bilateral optic nerves and the pituitary stalk appeared normal. A small incision was made on the superior surface of the chiasm and biopsy specimens were taken. The histological diagnosis was adenocarcinoma, which was consistent with the primary lung cancer. She received whole brain irradiation, resulting in mild improvement of her vision. Suprasellar metastatic tumors to the pituitary gland, pituitary stalk, and hypothalamus are well documented, but solitary metastasis within the optic chiasm should be considered as one of several differential diagnoses of suprasellar tumors, in patients with or without a history of treated cancer.
\end{abstract}

Key words: brain metastasis, optic chiasm, suprasellar tumor, lung cancer, optic glioma

\section{Introduction}

Metastasis to the pituitary gland, particularly from lung and breast cancers, is quite common, and usually manifests as diabetes insipidus, anterior pituitary dysfunction, visual field defects, headache, and ophthalmoplegia. ${ }^{5}$ Visual dysfunction due to suprasellar extension from pituitary metastases is known, whereas visual dysfunction due to metastasis directly to the optic chiasm is rare. We present a case of solitary metastasis within the optic chiasm.

\section{Case Report}

A 61-year-old woman presented with progressive deterioration of her vision for 2 months. She had undergone surgical excision and local radiotherapy for a lung adenocarcinoma one year previously. General physical examination was unremarkable. Neurological examination revealed a fully alert and cooperative patient except for the visual dysfunction. Ophthalmological examination revealed decreased visual acuity in both eyes and incomplete bitemporal hemianopsia (Fig. 1). Magnetic resonance (MR) imaging revealed a $1.0 \times 0.8 \mathrm{~cm}$ suprasellar mass separate from the pituitary gland that was slightly hypointense on $\mathrm{T}_{1}$-weighted imaging and hyperintense on $\mathrm{T}_{2}$ weighted imaging, with homogeneous enhancement after gadolinium administration (Fig. 2A-C). The preoperative MR imaging appearance was similar to that of optic glioma. The sellar turcica was normal, and the posterior lobe

Received June 2, 2009; Accepted July 17, 2009

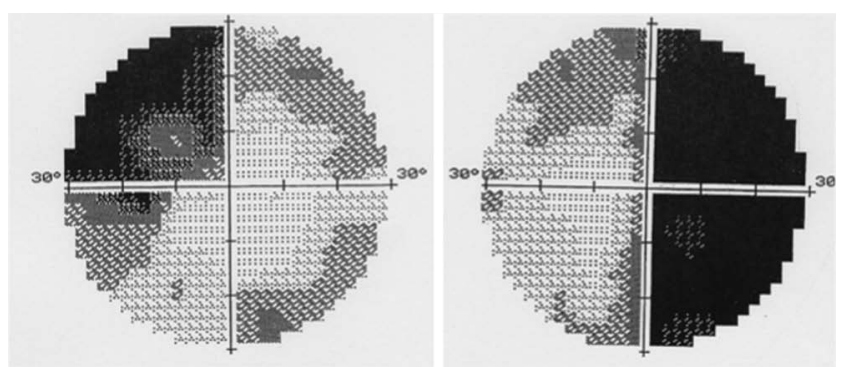

Fig. 1 Ophthalmological examination revealing incomplete bitemporal hemianopsia. Left: left eye, right: right eye.

of the pituitary gland remained hyperintense on sagittal $\mathrm{T}_{1}$-weighted imaging (Fig. 2D). Angiography showed no vascular-rich tumor. Her pituitary gland function was normal, except for mildly elevated serum prolactin level. Her level of carcinoembryonic antigen, a tumor marker of adenocarcinoma, was within the normal range. Our preoperative diagnosis was optic glioma or metastatic tumor.

Surgery was performed through a right trans-sylvian approach to explore the suprasellar lesion. The optic chiasm appeared markedly enlarged and showed hypervascularity over the surface (Fig. 3A). Both optic nerves and the pituitary stalk also appeared normal. A small incision was made in the superior surface of the chiasm, and biopsy specimens were taken (Fig. 3B). Histological examination revealed adenocarcinoma, indicating a metastatic tumor originating from non-recurrent lung cancer (Fig. 4). 

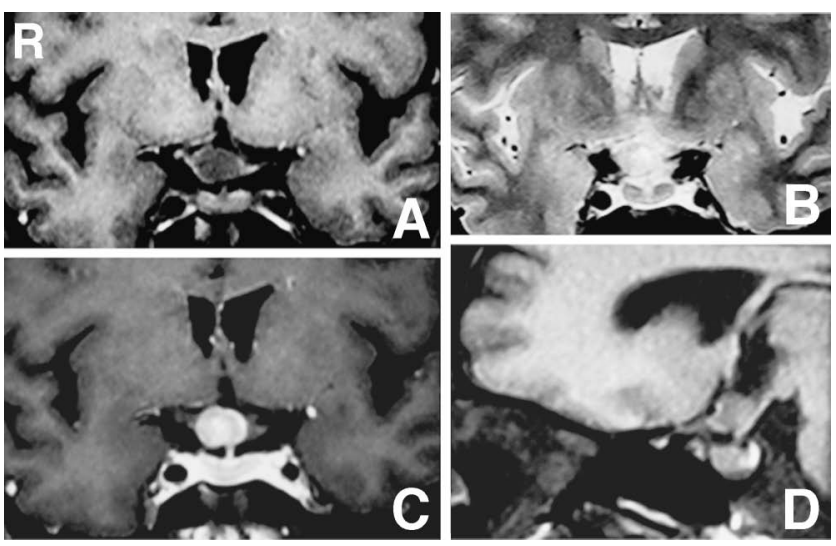

Fig. 2 (A) Preoperative coronal $\mathrm{T}_{1}$-weighted magnetic resonance (MR) image revealing a slightly hypointense suprasellar mass. (B) Coronal $\mathrm{T}_{2}$-weighted $\mathrm{MR}$ image revealing a hyperintense mass. (C) Coronal $\mathrm{T}_{1}$-weighted $M R$ image with gadolinium revealing a homogeneous enhanced mass in the suprasellar region. (D) Sagittal $T_{1}$-weighted MR image revealing a suprasellar mass distinct from the normal pituitary gland and stalk.
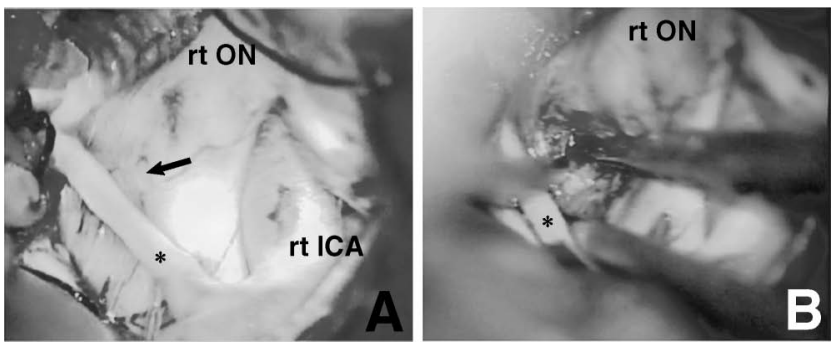

Fig. 3 (A) Intraoperative photograph revealing the markedly enlarged chiasm with hypervascularity over the surface (arrow) and normal optic nerve. (B) Intraoperative photograph showing a small incision made in the superior surface of the chiasm to take biopsy specimens. Asterisk: right anterior cerebral artery, rt ICA: right internal carotid artery, rt ON: right optic nerve.

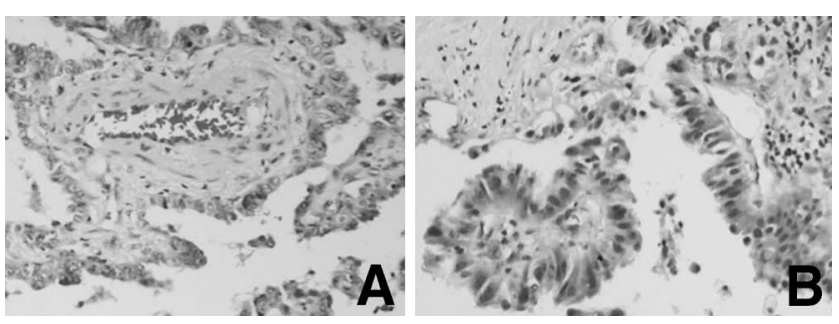

Fig. 4 (A) Photomicrograph of the lung cancer revealing papillary-like structures of the adenocarcinoma. Hematoxylin and eosin stain, $\times 100$. (B) Photomicrograph of the brain metastasis revealing the same pathological appearance as the lung cancer. Hematoxylin and eosin stain, $\times 100$.
Postoperatively, she did well with no new deficits. Postoperative MR imaging revealed a new small mass in the cerebellum. She received $30 \mathrm{~Gy}$ whole brain irradiation over 2 weeks, resulting in mild improvement of her vision. She did well without neurological deterioration for 18 months. However, visual acuity of her right eye gradually worsened, resulting in blindness due to regrowth of the optic chiasm metastasis without new lesions. She died of progressive systemic cancer 32 months after the diagnosis of brain metastasis.

\section{Discussion}

Only nine cases of metastasis to the optic chiasm have been reported: eight cases of metastasis indirectly to the optic chiasm, ${ }^{3,9,13,14)}$ and one case of metastasis directly to the optic chiasm. ${ }^{1)}$ All patients presented with visual deficits such as visual acuity deterioration and bitemporal hemianopsia, and two patients also had hormonal insufficiency. Optic chiasm metastasis was identified in three autopsy cases with multiple brain metastases, ${ }^{14)}$ and three cases of chiasmal syndrome due to suprasellar metastasis were reported without revealing the precise region of metastasis. ${ }^{3)}$ One case of optic chiasm metastasis was found expanding the infundibulum and infiltrating the hypothalamus, ${ }^{9}$ and one case of metastasis in the pituitary gland extending to the chiasm. ${ }^{13)}$ A solitary metastasis in the optic chiasm was identified during surgery. ${ }^{1)}$ Therefore, including our case, only two cases of solitary metastasis directly to the optic chiasm have been found (Table 1). In the present case, preoperative MR imaging demonstrated the solitary metastasis within the optic chiasm, which was confirmed during surgery.

Three mechanisms have been suggested for optic chiasm metastasis: hematogenous spread of tumors; infiltration of the chiasm by tumor cells from nearby structures, mainly the pituitary gland; and dissemination or drop metastasis from cerebrospinal fluid (CSF) spread. ${ }^{1,3,14)}$ Vascular support of the optic chiasm is derived primarily from the caudal side, via small branches of the anterior cerebral artery and the anterior communicating artery. The arteries are often in direct contact with the chiasm. ${ }^{8)}$ In the present case, MR imaging found no lesions in nearby structures such as the pituitary gland, stalk, and hypothalamus. The intraoperative findings also revealed that the pituitary stalk was normal, and the clinical symptoms included only visual dysfunction, and not diabetes insipidus or hypopituitarism. No meningeal carcinomatosis was observed during the clinical course. Therefore, hematogenous spread of tumor directly to the optic chiasm is the most likely mechanism of metastasis in our case. The same mechanism was considered in the previous case, ${ }^{1)}$ but infiltration from metastasis of nearby structures or dissemination from the CSF spread may have occurred in the other cases of optic chiasm metastasis. ${ }^{3,9,13,14)}$

The differential diagnoses of suprasellar lesion separate from the pituitary gland include craniopharyngioma, germ cell tumor, optic glioma, and metastatic tumor. Craniopharyngiomas have a bimodal peak of incidence, 


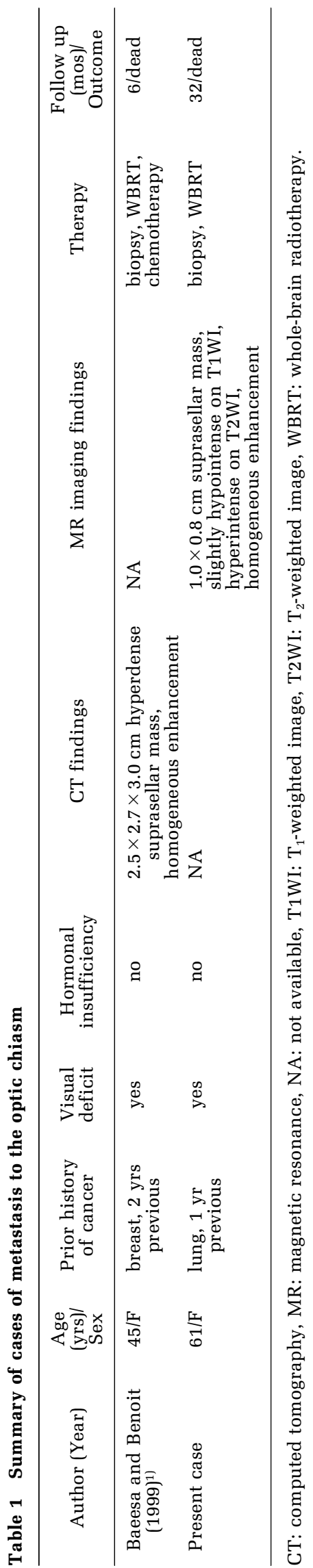

occurring predominantly in children. Central nervous system germ cell tumors occur most commonly in the second and third decades of life. ${ }^{6)}$ Glioma is frequently located in the chiasm or optic nerves, and approximately $90 \%$ of these optic gliomas occur in children. Adult onset optic gliomas are markedly different from the optic gliomas of childhood, being typically malignant, rapidly progressive, and demonstrate uniform enhancement and expansion of the involved optic pathway. ${ }^{4,7)}$ Other rare differential diagnoses in aged patients include lymphoma and ectopic pituitary adenoma. ${ }^{10,16)}$ These lesions also appear as homogeneously enhanced mass on MR imaging. In our case, the optic chiasm metastasis was slightly hypointense on $\mathrm{T}_{1}$-weighted imaging, hyperintense on $\mathrm{T}_{2}$-weighted imaging, and homogeneously enhanced. Therefore, only the neuroimaging findings cannot differentiate optic chiasm metastasis from the other differential diagnoses.

For patients with solitary brain metastasis, a radical treatment approach involving surgical resection or stereotactic radiosurgery, followed by whole brain radiotherapy is recommended. ${ }^{12}$ If a tumor invades the optic nerve, fractionated radiation is the best treatment because the tolerance of the optic apparatus is the limiting factor. ${ }^{2}$ Surgical resection of optic chiasm metastasis is thought to be challenging and unsafe because of the significant morbidity. ${ }^{1)}$ Chemotherapy is controversial. In general, chemotherapy is effective for brain metastases of small-cell carcinoma and germ cell tumors. ${ }^{11)}$ Recently, gefitinib, a tyrosine kinase inhibitor of the epidermal growth factor receptor, has shown promising potential in palliative therapy for patients with brain metastasis of lung adenocarcinoma. ${ }^{15)}$ In our case, mild improvement of the patient's visual acuity was obtained by whole brain radiotherapy following surgical biopsy, and maximum quality of life was maintained for a long period. The therapeutic strategy in the present case seemed to be appropriate.

The present rare case of solitary metastasis within the optic chiasm shows that metastasis should be considered as one of several differential diagnoses of suprasellar tumors, in patients with or without a history of treated cancer. Preoperative neuroimaging diagnosis of suprasellar lesions is difficult in adults, so surgical biopsy to establish the pathology is essential.

\section{References}

1) Baeesa SS, Benoit BG: Solitary metastasis of breast carcinoma in the optic chiasm. Br J Neurosurg 13: 319-321, 1999

2) Borden JA: Treatment of tumors involving the optic nerves and chiasm. Semin Ophthalmol 17: 22-28, 2002

3) Cohen MM, Lessell S: Chiasmal syndrome due to metastasis. Arch Neurol 36: 565-567, 1979

4) Dinh TT, Wang YY, Rosenfeld JV, Cherny M: Glioblastoma of the optic chiasm. J Clin Neurosci 14: 502-505, 2007

5) Fassett DR, Couldwell WT: Metastases to the pituitary gland. Neurosurg Focus 16(4): E8, 2004

6) Freda PU, Post KD: Differential diagnosis of sellar masses. Endocrinol Metab Clin North Am 28: 81-117, 1999

7) Miyamoto J, Sasajima H, Owada K, Mineura K: Surgical decision for adult optic glioma based on [18F]fluorodeoxyglu- 
cose positron emission tomography study. Neurol Med Chir (Tokyo) 46: 500-503, 2006

8) Ove R, Kelman S, Amin PP, Chin LS: Preservation of visual fields after peri-sellar gamma-knife radiosurgery. Int J Cancer 90: 343-350, 2000

9) Schwartz WJ, Busis NA, Hedley-Whyte ET: A discrete lesion of ventral hypothalamus and optic chiasm that disturbed the daily temperature rhythm. J Neurol 233: 1-4, 1986

10) So G, Takeshita T, Morofuji Y, Iseki M, Hayashi T, Matsuo T, Suyama K, Nagata I: [Nonfunctioning suprasellar ectopic pituitary adenoma. A case report]. No Shinkei Geka 36: 1121-1125, 2008 (Jpn, with Eng abstract)

11) Soffietti R, Ruda R, Mutani R: Management of brain metastases. J Neurol 249: 1357-1369, 2002

12) Taimur S, Edelman MJ: Treatment options for brain metastases in patients with non-small-cell lung cancer. Curr Oncol Rep 5: 342-346, 2003

13) Takahashi M, Kinouchi H, Sasajima T, Suzuki A, Mizoi K: [A case of metastatic suprasellar tumor with edema along the optic pathway]. No Shinkei Geka 31: 775-779, 2003 (Jpn, with Eng abstract)

14) Takahashi T, Murase T, Isayama Y: [Clinicopathological findings in the chiasmal region with reference to metastatic carcinoma cases (author's transl)]. Nippon Ganka Gakkai Zasshi 83: 229-234, 1979 (Jpn, with Eng abstract)

15) Wu C, Li YL, Wang ZM, Li Z, Zhang TX, Wei Z: Gefitinib as palliative therapy for lung adenocarcinoma metastatic to the brain. Lung Cancer 57: 359-364, 2007

16) Zelefsky JR, Revercomb CH, Lantos G, Warren FA: Isolated lymphoma of the anterior visual pathway diagnosed by optic nerve biopsy. J Neuroophthalmol 28: 36-40, 2008

Address reprint requests to: Atsushi Arai, M.D., Department of Neurosurgery, Kobe University Graduate School of Medicine, 7-5-2 Kusunoki-cho, Chuo-ku, Kobe 650-0017, Japan. 